Ann. Génét. Sél. anim., 1981, 13 (2), 177-180

NOTE

\title{
Localisation des organisateurs nucléolaires dans les chromosomes du Cheval domestique
}

\author{
(Equus caballus)
}

\author{
E.P. CRIBIU \\ I.N.R.A., Laboratoire de Cytogénétique, U.N.C.E.I.A. \\ Centre de Recherches zootechniques \\ F 78350 Jouy-en-Josas
}

\begin{abstract}
Résumé
Les régions organisatrices de nucléoles (R.O.N.) du Cheval domestique (Equus caballus L.) mises en évidence par la coloration à l'argent ont été localisées à l'extrémité des bras courts de la première paire autosomale A1 et dans la constriction secondaire de la paire E29. Des associations entre les chromosomes impliqués ont été observées.
\end{abstract}

Le nombre de chromosomes diploïde $2 \mathrm{n}=64$ du Cheval domestique a été définitivement établi par Rothfels et al. (1959). Les autosomes ont été rangés dans un idiogramme par De Giovanni et al. (1979) en 5 groupes : A, B, C, D et E, en fonction de leur morphologie. En coloration classique une des 18 paires d'acrocentriques possède une constriction secondaire sur les bras longs, près du centromère (Trujillo et al., 1962 ; Hsu \& BenirschKe, 1967 et Bornstein, 1967). Il s'agit, selon la convention, ci-dessus évoquée, de la paire de petits acrocentriques E 29. Quelques auteurs (Trujillo et al., 1962 et Hsu \& BENIRSCHKe, 1967) ont également observé un satellite à l'extrémité des bras courts de la première paire des grands submétacentriques $\mathbf{A} 1$.

Les relations entre constrictions secondaires et régions des organisateurs du nucléole (R.O.N.) qui ont déjà été mises en évidence dans d'autres systèmes génétiques, laissent à penser que ces deux paires de chromosomes pourraient contenir des sites de synthèses du A.R.N. ribosomal chez le Cheval domestique.

Les régions organisatrices du nucléole ont déjà été observées dans d'autres espèces. par des techniques différentes : hybridation in situ (Henderson et al., 1972), marquage N (FunaKi et al., 1975) et coloration à l'argent (Howell et al., 1975; GoodPASTURE \& Bloom, 1975 et Bloom \& Goodpasture, 1976). Nous nous sommes proposés ici de les localiser chez le Cheval domestique. 
Les préparations chromosomiques nécessaires ont été obtenues à partir de cultures à court terme de sang périphérique de 22 étalons phénotypiquement normaux, selon la méthode de Moorhead et al. (1960). Nous avons utilisé la coloration à l'argent mise au point par PATHAK \& ELDER (1980).

Dans toutes les métaphases étudiées, un marquage sur les paires autosomales A1 et E29 a été observé. Les précipités d'argent se localisent en effet en quantité variable d'un animal à l'autre dans la région terminale des bras courts du chromosome submétacentrique $\mathrm{A} 1$ et sur les bras longs, près du centromètre, du chromosome E29 (Fig. 1). Dans un petit nombre de métaphases appartenant à deux animaux, nous avons également observé un marquage près du centromère, sur un homologue d'une paire d'acrocentrique de taille moyenne.

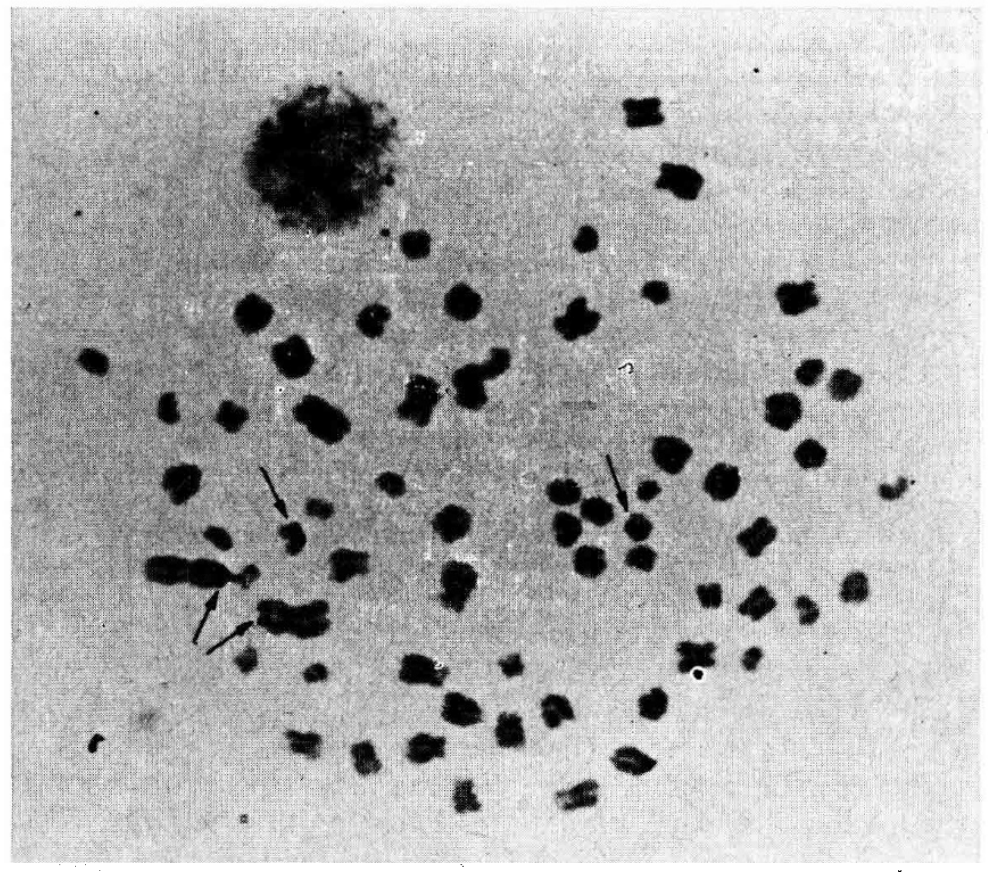

FIG. 1

e

Métaphase colorée à l'argent

(Les chromosomes organisateurs nucléolaires sont marqués par des flèches)

Metaphase of a 64, XY horse showing Ag-N.O.R.s on two pairs, both chromosomes A1 and both chromosomes E29

(The arrows indicate chromosomes $A 1$ and E29)

De nombreuses associations entre les homologues de la paire A1 et les chromosomes A1 et E29 ont aussi été remarquées (Fig. 2a et 2b). 

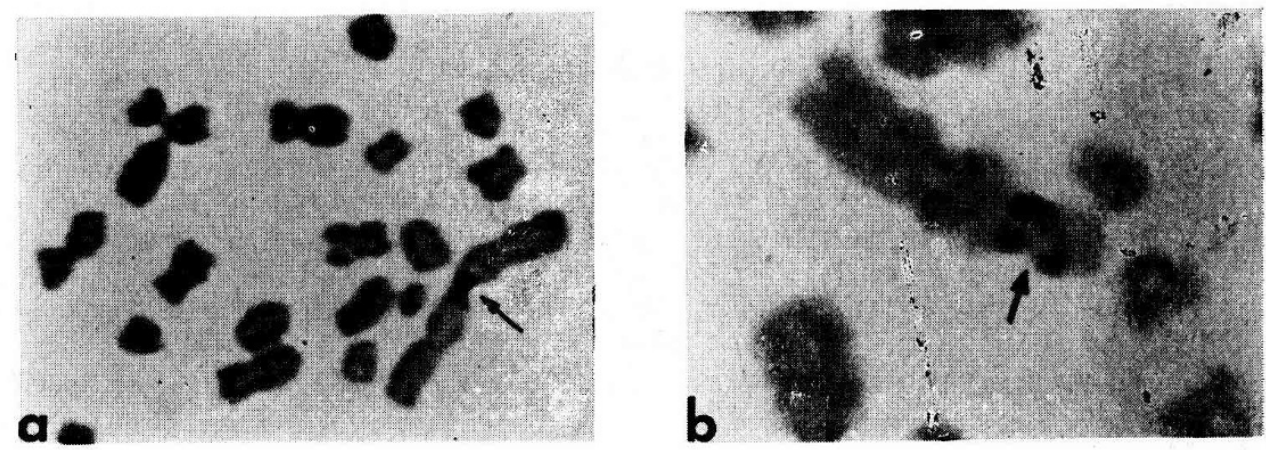

FIG. 2

Association des chromosomes organisateurs nucléolaires

a) association des homologues de la paire A1

b) association des chromosomes A1 et E29

Involvement of each type of nucleolus organizer chromosome in association complexes

a) Two Al associations

b) A1 and E29 associations

Il découle de ces observations que les constrictions secondaires se trouvant sur les paires A1 et E29 correspondent bien à des sites de synthèses de l'A.R.N. ribosomal. Cependant, les techniques de coloration à l'argent ne marquent pas toutes les régions qui contiennent les gènes codant pour l'A.R.N.r, car leur localisation dépend probablement du nombre de gènes présents et actifs dans une R.O.N. particulière. Il faut sans doute qu'un nombre minimum de copies d'A.D.N.r soit présent pour qu'une observation microscopique les révèle. Les régions qui en possèdent relativement peu peuvent apparaître inactives et, de ce fait, ne pas être révélées par la coloration à l'argent.

Selon certains auteurs les associations chromosomiques observées à la métaphase pourraient entraîner la formation d'anomalies chromosomiques. Ainsi les chromosomes organisateurs nucléolaires pourraient, par ce phénomène, être les plus susceptibles de subir certains remaniements comme par exemple des translocations.

Reçu pour publication en mai 1981.

\section{Summary}

\section{Nucleolus organizer regions in domestic horse (Equus caballus L.)}

The nucleolus organizer regions (N.O.R.s) of domestic horse (Equus caballus L.) as shown by silver staining, are located at the telomere of the short arms of the longest autosome $\mathrm{A} 1$ and in the secondary constriction of the small acrocentric one E29 


\section{Références bibliographiques}

Bloom S.E., Goodpasture C., 1976. An improved technique for selective silver staining of nucleolar organizer regions in human chromosomes. Hum. Genet., 34, 199-206.

Bornstein S., 1967. The genetic sex of two intersexual horses and some notes on the karyotype of normal horses. Acta Vet. Scand., 8, 291-300.

De Grovanni A., Molteni L., Succi G., Castiglioni M., Cribiu E.P., 1979. The idiogram of the domestic horse (Equus caballus L.). Caryologia, 32, 215-222.

Funaki K., Matsui S., SASaKi M., 1975. Location of nucleolar organizers in animal and plant chromosomes by means of an improved N-banding technique. Chromosoma, 49, 357-370.

Goodpasture C., Bloom S.E., 1975. Visualysation of nucleolar organizer regions in mammalian chromosomes using silver staining. Chromosoma, 53, 37-50.

Henderson A.S., Warburton D., Atwood K.C., 1972. Location of $r$ D.N.A. in the human chromosome complement. Proc. natl. Acad. Sci. (Wash.), 69, 3394-3398.

Howell W.M., Denton T.E., Diamond S.R., 1975. Differential staining of the satellite regions of human acrocentric chromosomes. Experientia, 31, 260-262.

Hsu T.C., BenirschKe K., 1967. An atlas of mammalian chromosomes. Springer Verlag, 1, 34.

Moorhead P.S., Nowell P.C., Mellman W.J., Battips D.M., Hundgerford D.A., 1960. Chromosome preparations of leucocytes cultured from human periphiral blood. Exp. Cell Res., 20, 613-616.

Pathak S., Elder F.F.B., 1980. Silver stained accessory structures on human sex chromosomes. Hum. Genet., 54, 171-173.

Rothfelds K.M., Axelrad A.A., Siminovitch L., McCulloch E.A., Parker R.C., 1959. The origin of altered cell lines from mouse, monkey and man as indicated by chromosome and transplantation studies. Can. Cancer Conf. (R.W. Bigg, editor, Academic Press, Inc., New York), 3, 189-214.

Trujillo J.M., Stenius C., Christian L.C., Ohno S., 1962. Chromosomes of the horse, the donkey and the mule. Chromosoma, 13, 243-248. 